\title{
Giant gastric trichobezoar in a teenage girl with trichotillomania
}

\author{
Oana-Maria Rosu', Flavia Luca' ${ }^{2}$, Ana-Maria Scurtu ${ }^{3}$, Anamaria Ciubara ${ }^{4}$, \\ Smaranda Diaconescu ${ }^{5}$, Marin Burlea ${ }^{6}$ \\ 1"Sf. Maria" Clinical Emergency Children's Hospital, lasi, Romania \\ ${ }^{2}$ Radiology Clinic, "Sf. Maria” Clinical Emergency Children's Hospital, lasi, Romania \\ ${ }^{3}$ Pediatric Surgery Clinic, "Sf. Maria" Clinical Emergency Children's Hospital, Iasi, Romania \\ 4"Dunarea de Jos" University, "Elena Doamna" Psychiatry Hospital, Galati, Romania \\ 5"Titu Maiorescu" University, Faculty of Medicine, Pediatric Gastroenterology Clinic - "Sf. Maria" \\ Clinical Emergency Children's Hospital, Iasi, Romania \\ 6"Gr. T. Popa" University of Medicine and Pharmacy, Iasi, Pediatric Gastroenterology Clinic - \\ "Sf. Maria" Clinical Emergency Children's Hospital, Iasi, Romania
}

\begin{abstract}
Introduction. A bezoar is a conglomerate comprising partially digested or undigested matters, impacted in the stomach. Trichobezoars (agglomerations of hair) occur most frequently in patients with mental disorders (trichotillomania) and represent a rare pathology that can entail diagnostic and therapeutic difficulties.

Material and method. We are reporting a gastric trichobezoar case in 12 years old female patient with no pathological personal history, admitted for epigastric pain and food vomiting. Clinical examination revealed an epigastric formation of approx. $5 / 3 \mathrm{~cm}$, rigid, immobile and adhering to the deep structure, while laboratory tests revealed a mild microcytic hypochromic anemia. Upper gastrointestinal endoscopy provided visualization of a giant trichobezoar of approximately $17 \mathrm{~cm}$, visible from the subcardial region down to the antral region, hooked by one hair onto an area with a pseudopolypoid inflammatory aspect located in the prepyloric region. The patient was referred to the pediatric surgery department, as the size of the trichobezoar did not allow for its endoscopic extraction. The initial attempt for laparoscopic extraction was unsuccessful and required a minimal left subcostal laparotomy, followed by gastrotomy with evacuation of the gastric trichobezoar. The postsurgical evolution was favorable.

Discussions. Despite its low prevalence in humans, mortality can sometimes reach $30 \%$ in certain cases due to the bleeding, obstruction, peritonitis or ultimately the gastrointestinal perforations that could occur. Various endoscopic methods were reported for bezoar extractions, such as shock wave lithotripsy, removal via aspiration using a large gauge endoscope, extraction with polypectomy snare and biopsy forceps.

Conclusion. The rareness of this pathology in the pediatric population requires a very careful differential diagnosis. The therapeutic difficulties result from the size of the trichobezoar, while the prognosis depends on the underlying psychiatric pathology.
\end{abstract}

Keywords: trichobezoar, trichotillomania, endoscopy

\section{INTRODUCTION}

Bezoars are conglomerates of undigestible materials such as hair, which are usually located in the stomach. Bezoars have been reported in the literature as early as the $12^{\text {th }}$ century, initially being described in India. Owing to their rareness and their unclear pathogenesis, bezoars were ascribed special properties during those times. The term Bezoar comes from either the Arab "Bedzehr", the Persian "Padzahr" or the Hebrew "Beluzaar", all of which have the meaning of antidote. Based on their contents, there are four categories of bezoars: phytobezoar (comprising vegetal fibers), trichobezoar (hair), lactobezoar (dairy), as well as miscellaneous (medication, sand, absorbent paper) $(1,2)$. The most frequent type of bezoar is phytobezoar, which is composed of cellulose. Trichobezoar is the second most common type, most often caused by trichophagia (the ingestion of hair), combined with a mental disorder (trichotillomania). Trichotillomania, the compulsive urge to pull one'shair, was first de- 
scribed by Hallopeau in 1889 , and the first trichobezoar case was reported one century earlier, in 1792 , by Baudamant $(3,4)$. This pathological condition primarily affects young women, with or without known psychiatric disorders.

\section{CASE REPORT}

A twelve years old female patient without any significant pathological personal history was admitted to the clinical department of pediatric gastroenterology, where she was referred from a regional hospital, with suspected gastric tumor. Upon admission, the patient presented with epigastric pain and food vomiting. The family inquiry revealed no conflicts within the family which could generate psychological stress. Palpation in the epigastric region revealed a formation of approx. 5/3 $\mathrm{cm}$, rigid, immobile and adhering to the deep structure. Paraclinical examinations revealed a mild microcytic hypochromic anemia, without any other pathological changes. Abdominal ultrasound showed a hyperechogenic area, with a posterior shadow cone, located in the epigastric and umbilical region (Fig.1).

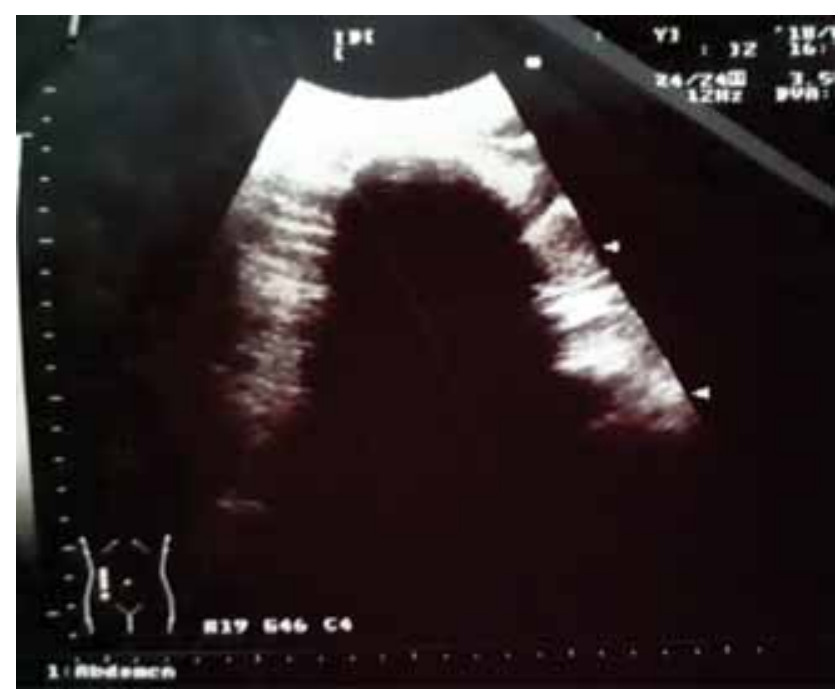

FIGURE 1. Abdominal ultrasound - hyperechogenic area, with posterior shadow cone

Endoscopy provided visualization of a mass of approx. $17 \mathrm{~cm}$, visible from the subcardial region down to the antral region, hooked by one hair onto an area with a pseudopolypoid inflammatory aspect located in the prepyloric region (Fig. 2).

The patient underwent a neuropsychiatric consult and was diagnosed with trichotillomania; recommendations included psychologic counselling and risperidone $0.5 \mathrm{mg} /$ day for 30 days. Abdominal CT findings include: well-defined inhomogeneous

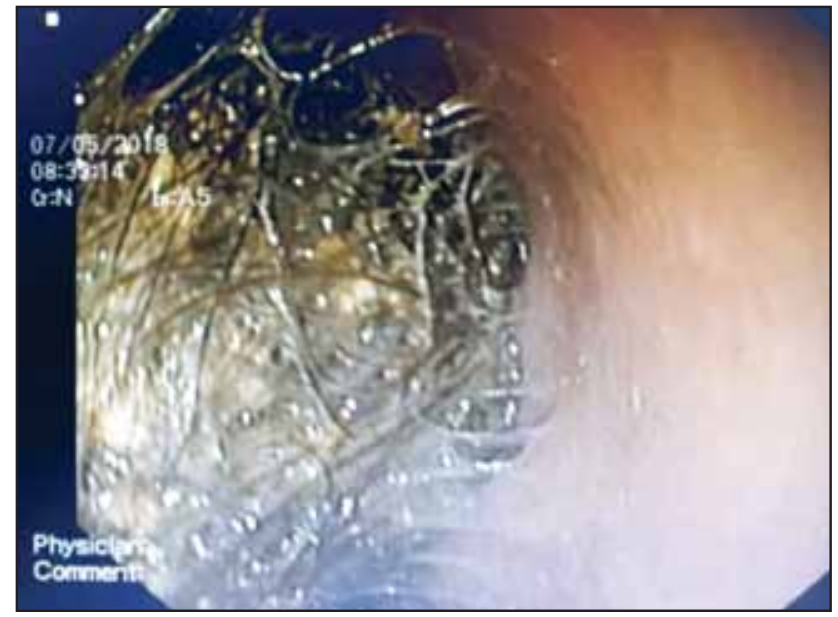

FIGURE 2. Endoscopic image of the trichobezoar

soft tissue mass with air inclusions, visible in the gastric lumen, with solid-type densities, without contrast medium uptake, measuring approx. 109/39 $\mathrm{mm}$ and located in the corporeal-antral region down to the pyloric canal, dilated stomach with mild circumferential wall thickening, antral gastric region with an anteroposterior diameter of $60 \mathrm{~mm}$ and circumferential pyloric wall thickening of approx. 6.4 mm (Fig. 3).

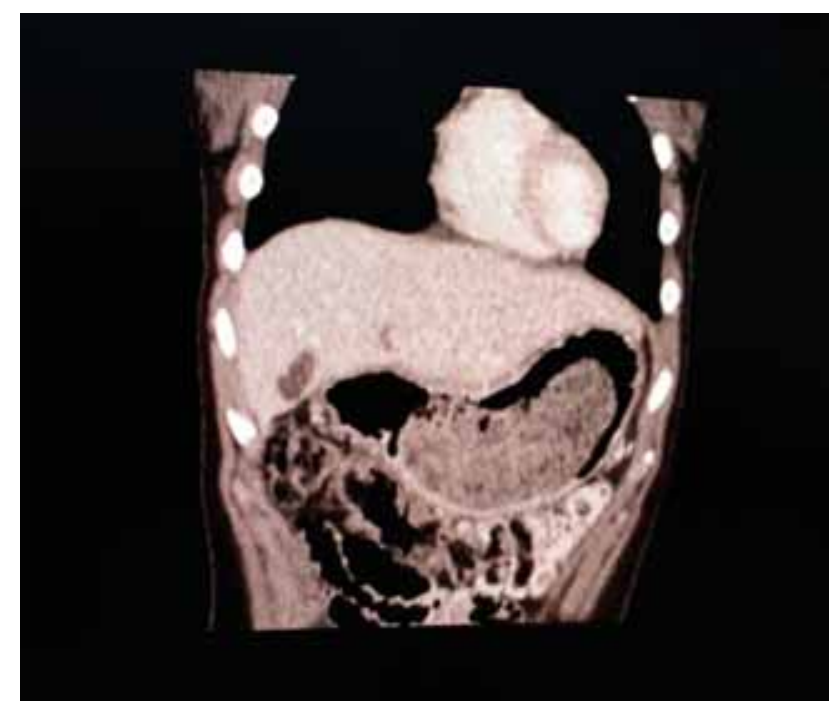

FIGURE 3. Abdominal CT - Inhomogeneous soft tissue mass with air inclusions

The size of the trichobezoar did not allow for its endoscopic extraction, which is why the patient was transferred to the pediatric surgery department. The initial plan was to extract the bezoar laparoscopically but was subsequently switched to classic surgery (minimal left subcostal laparotomy, followed by gastrotomy with evacuation of the gastric trichobezoar) (Fig. 4).

The postsurgical evolution was favorable. The teenage girl was discharged 10 days after surgery, 


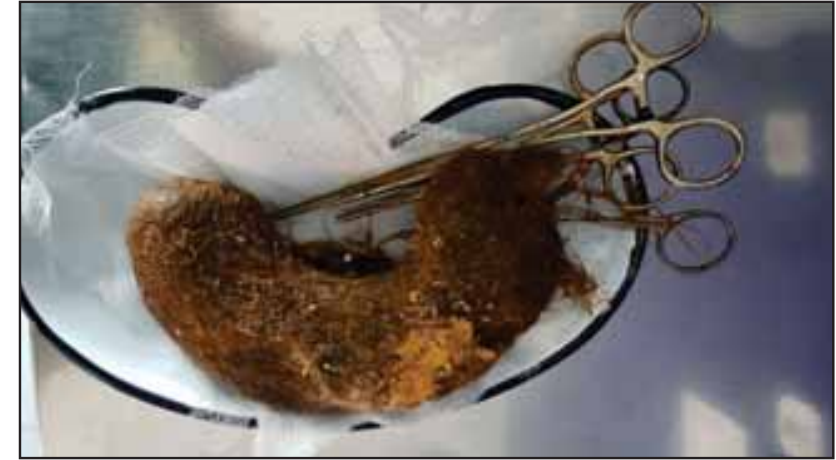

FIGURE 4. Gastric trichobezoar - surgical piece

remaining under the dynamic monitoring of the multidisciplinary team (gastroenterologist, surgeon and neuropsychiatrist).

\section{DISCUSSIONS}

Trichobezoars are the most frequent type of bezoar occurring in the young population. In DeBakey and Ochsner's classic review, $80 \%$ of cases were found in patients under the age of 30 . The ingestion of hair or fibers from carpets or clothing results in the formation of a mass that subsequently becomes blocked in the folds of the gastric mucosa, as the slippery surface prevents the propulsion triggered by peristaltic waves (5). Trichobezoars are unique and generally takes the shape of the stomach (6-8). The mucus covering it confers it a glossy, shiny surface, while the breakdown and fermentation of fats confer its specific fetid odor.Owing to the peptic digestion of the hairs, the trichobezoar most often takes on a black shade, irrespective of the initial color of the ingested hair $(9,10)$

Literature studies show that trichobezoars are frequently found in the population under the age of 30 , but it has a higher prevalence in female patients under the age of 20. Common reported symptoms include abdominal pain, nausea and vomiting, bloating, early satiety, weight loss, diarrhea or constipation. However, there are no characteristic signs or pathognomonic symptoms for bezoars. Our case was consistent with these findings, as the patient presented a non-specific symptomatology. Trichobezoars that evolve over extensive periods of time can cause complications, including the occurrence of anemia, hematemesis, gastric ulcer, bowel obstruction, perforation, peritonitis, pancreatitis, obstructive jaundice, malabsorption syndrome, invaginations and appendicitis (11). Upper GI endoscopy is the gold standard for diagnosis, as it ensures the direct visualization of the bezoar and enables the subsequent therapeutic approach. Endoscopic ther- apy can only be effective for bezoars containing vegetal matter (phytobezoars) and milk (lactobezoars), which have smaller sizes; this is less likely to be effective for trichobezoars, particularly larger ones $(>20 \mathrm{~cm})$. Different rates of efficiency are reported for various other methods, such as extracorporeal litotrition with intragastric administration of enzymes (pancreatic lipase, cellulase) and medication (metoclopramide, acetylcysteine) (12).

Trichotillomania is defined as self-inflicted recurrent hair loss. The prevalence of trichotillomania during the lifetime is estimated at around 0.6$4.0 \%$ of the global population (13). Trichotillomania is diagnosed in all age groups; the onset is more frequent during preadolescence and young adulthood, with a mean onset age between 9 and 13 years old, and a notable peak between 12 and 13 years. In preschool age children, trichotillomania is considered benign. For these children, hair pulling often begins in times of psychosocial stress and it almost never continues with age. Gender distribution is approximately equal among preschool children; there appears to be a female prevalence among preadolescents (70\%-93\%), while among young adults there is a 3 to 1 female-to-male ratio $(14,15)$.

According to D.S.M.-IV-2003, the following diagnostic criteria were determined for this disorder: a. recurring inability to resist impulses to pull out one's own hair, resulting in the significant loss thereof; $b$. increasing sense of tension right before pulling out the hair; c. sense of pleasure, gratification or relief when pulling out the hair; $d$. the behavior causes suffering or significant damage in terms of social functioning; $d$. there is no association with a preexisting inflammation of the skin and does not occur as a response to delirious ideas or hallucinations $(16,17)$. Patients try to cover this behavior or to conceal the affected area of the scalp, with an objective examination revealing short, torn strands mixed with normal hair growth. The trichotillomania diagnosis is suspected when the compulsive hair pulling behavior is not justified by a general medical condition (e.g. a dermatological condition) or another psychiatric disorder (such as delirium or as a result of hallucinations), and it can be confirmed via upper gastrointestinal endoscopy. Psychiatric or psychological evaluation is essential given that underlying emotional stress is often a triggering factor for trichophagia or trichotillomania as observed in affected patients.

With respect to the treatment of a bezoar, this should include the removal of the mass and essentially preventing recurrences by approaching the 
physical or emotional causes underlying the onset of this pathology. Depending on its consistency, size and location, removal of the bezoar can be performed by endoscopy or surgical intervention gastrotomy by superior median laparotomy. Although there is no medication-based treatment approved specifically for trichotillomania, some drugs can help control certain symptoms, such as antidepressants (e.g. clomipramine). Other drugs that research suggests could have certain benefits contain $\mathrm{N}$-acetylcysteine, an aminoacid influencing the neurotransmitters linked to the mood, and olanzapine, a mild antipsychotic (18-20). Behavioral strategies that are effective in the treatment for trichotillomania may include: self-monitoring, cognitive-behavioral therapy, relaxation classes, psychotherapy, hypnosis. Prognosis can vary depending on the associated psychiatric pathology, which is why some people can have a symptomatology that continues for decades, while other times the evolution of this disorder is marked by remission and relapse phases.

In our case, the diagnosis was confirmed via upper gastroeintestinal endoscopy. Trichobezoar ex-

\section{REFERENCES}

1. Lee J. Bezoars and foreign bodies of the stomach. Gastrointest Endosc 1996; 6: 605-619.

2. Feldman, M., Friedman, L.S., Brandt, L.J. Foreign bodies, bezoars, and caustic ingestions. In: Sleisenger and Fordtran's Gastrointestinal and Liver Disease. 9th ed. Philadelphia, PA: Elsevier; 2010.

3. Faria A.P., Silva I.Z., Santos A., Avilla S.G.A., Silveria A.E. The Rapunzel syndrome - a case report: trichobezoar as a cause of intestinal perforation (in Portuguese). J Pediatria (Rio J) 2000; 76: 83-86.

4. Santos T., Nuno M., Joao A. et al. Trichophagia and trichobezoar: Case report. Clin Pract Epidemiol Ment Health. 2012; 8:43-45.

5. DeBakey M., Ochsner A. Bezoars and concretions: a comprehensive review of the literature with analysis of 303 collected cases and a presentation of 8 additional cases. Surgery. 1939; 5: 132-160s

6. Pace A.P., Fearne C. Trichobezoar in a 13 year old male: a case report and review of literature, Malta Med J 2003; 15: 39-40.

7. Gorter R.R., Kneepkens C.M., Mattens, E.C. et al. Management of trichobezoar: Case report and literature review. Pediatr Surg Int. 2010; 26:457-463.

8. Salem M., Fouda R., Fouda U., Maadawy M.E., Ammar H. Rapunzel and pregnancy. South Med J. 2009; 102(1):106-7.

9. O'Sullivan M.J., McGreal G., Walsh J.G. Trichobezoar. J R Soc Med 2001; 94: 68-70.

10. Singla S.L., Rattan K.N., Kaushik N., Pandit S.K. Rapunzel syndrome: A case report. Am J Gastroenterol. 1999; 94(7):1970-1971.

11. Wadlington W.B., Rose M., Holcomb G.W. Complications of trichobezoars: a 30-year experience. South Med J X5:1020-1022, 1992. traction was performed using the traditional approach, via gastrotomy with minimal left subcostal laparotomy. Psychiatric or psychological evaluation is essential given that underlying emotional stress is most often the triggering factor for trichophagia or trichotillomania, as well as in terms of prognosis. Our patient was referred to a psychiatric unit for evaluation and specialized treatment.

\section{CONCLUSIONS}

Trichobezoars should be considered as a potential diagnosis in pediatric patients with a history indicative of trichophagia and a palpable epigastric mass. Diagnosis is made via upper gastrointestinal endoscopy, which sometimes also provides the therapeutic approach. The management of this pathology often requires surgical intervention. The multidisciplinary approach is essential, as the majority of these patients present with an underlying psychiatric disorder.

Conflict of interest: none declared Financial support: none declared

12. Groenewald C.B., Smoot R.L., Farley D.R. A football-sized gastric mass in a healthy teen. Contemp Surg 2006; 62:531-534.

13. Huynh M., Gavino A.C., Magid M. (June 2013). "Trichotillomania". Semin Cutan Med Surg. 32 (2): 88-94.

14. Sah D.E., Koo J., Price V.H. Trichotillomania. Dermatol Ther. 2008; 21: 13-21. PMID: 18318881

15. Chamberlain S.R., Menzies L., Sahakian B.J., Fineberg N.A. (April 2007). "Lifting the veil on trichotillomania". Am J Psychiatry. 164 (4): 568-74.

16. Chamberlain S.R., Odlaug B.L., Boulougouris V., Fineberg N., Grant J.E. Trichotillomania: neurobiology and treatment. Neurosci Biobehav Rev 2009; 33 (6) 831-842.

17. Lafleur D.L., Pittenger C., Kelmendi B., Gardner T., Wasylink S., Malison R.T., Sanacora G., Krystal J.H., Coric V. N-acetylcysteine augmentation in serotonin reuptake inhibitor refractory obsessivecompulsive disorder. Psychopharmacology (Berl)2006; 184 (2) 254-256.

18. First M.B., Spitzer R.L., Gibbon M., Williams J.B.W. Structured Clinical Interview for DSM-IV-TR Axis I Disorders, Research Version, Non-Patient Edition (SCID-I/NP). New York Biometrics Research, New York State Psychiatric Institute, 2002.

19. Rothbaum B.O., Ninan P.T. The assessment of trichotillomania. Behav Res Ther, 1994; 32 (6) 651- 662.

20. Christenson G.A., Mansueto C.S. Trichotillomania: descriptive characteristics and phenomenology. Stein DJ, Christianson GA, Hollander E. Trichotillomania. Washington, DC American Psychiatric Press, 1999;1-41. 